\title{
LA FILOSOFÍA DE LA ADMINISTRACIÓN DE PETER DRUCKER
}

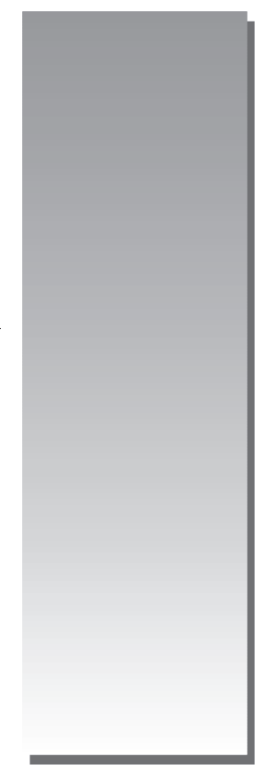

Juan Puell Palacios (*)

E-mail: juanpuell@hotmail.com

\section{RESUMEN}

Peter Drucker, al plantear una filosofía sobre las organizaciones, reflexiona ampliamente sobre el dinero como un recurso vital para los negocios. Es recomendable trabajar con dinero ajeno, pero el mismo tiempo éste debe ser devuelto para honrar el compromiso hecho y así poder ser sujeto de crédito en el mercado financiero.

Hace un enfoque sobre la gestión en las organizaciones la misma que, según su concepto, está relacionada con la gente que tiene la responsabilidad de llevarlas al éxito. Aquí Peter Drucker establece una relación indesligable entre el negocio y el cliente porque su satisfacción depende de los cambios que la gestión pueda hacer a su favor, para así mantenerlo con lealtad y convicción.

Cuando Peter Drucker analiza el conocimiento lo considera como un vínculo entre aprendizaje y enseñanza, que permite hacerlo más productivo cuando se aplica. Considera también que el conocimiento de hoy es obsoleto para el mañana por la dinámica de los cambios científicos y tecnológicos. Finalmente considera a las personas, reconociendo sus fortalezas y sus potencialidades, y propone que las organizaciones inviertan en el desarrollo de sus capacidades, habilidades y cualidades humanas.

Todos los administradores profesionales debemos afrontar el reto de crear nuestra propia filosofía para que las organizaciones empresariales apliquen los conocimientos para el bienestar de la sociedad en su conjunto.

Palabras clave: Filosofía, organizaciones

\section{ABTRACT}

In Peter Drucker's philosophy on the organizations he thinks widely concerning money, as a vital resource for the business, is like the deep thought for the philosophers. It is advisable to work with foreign money, but the same time the commitment must honor to return it and this way to be able to be a credit subject on the financial market.

An approach does on the management in the organizations, the same one that, according to his concept it belongs related to the people who has responsibility of taking them to the success. Here Peter Drucker establishes a relation unbreakable between the business and the client.

(") Licenciado en Administración. Profesor Asociado UNMSM. Director de la Escuela Académica Profesional de Administración de la Facultad de Ciencias Administrativas de la UNMSM. 
Customer's satisfaction depends on the changes that the management could make to yours favor and this way support it with loyalty and conviction.

When Peter Dancker analyzes the knowledge he considers it to be a link between learning and education to make it more productive when it is applied. He thinks that the today knowledge is obsolete for tomorrow for the dynamics of the scientific and technological changes. Finally he considers the persons, recognizing theirs fortresses, potentials and proposes that the organizations should invest in the development of theirs capacities, skills and human qualities. All the managers must confront the challenge of creating our own philosophy in order that the managerial organizations apply the knowledge for the well-being of the throughout society.

Key words: Philosophi, organizations

Existen valiosos estudios sobre la filosofía de la ciencia, filosofía de la tecnología, filosofía del arte, de la historia, de la economía, etc. En cambio existen muy pocos estudios e investigaciones sobre la filosofía de la administración o filosofía de la gerencia. No soy filósofo pero tengo tiempo para reflexionar en este campo porque me dedico a hablar, leer y escribir sobre este tema tan igual como hacen los filósofos, con la diferencia de que estos lo hacen en un nivel un poco distinto al lenguaje cotidiano.

En el libro "Enseñanzas de Peter Drucker" se plantean algunas conceptualizaciones relacionadas con la filosofía de la gerencia que pueden servir a los administradores para realizar mayores estudios e investigaciones. Peter Drucker nos hace reflexionar sobre la eficiencia de las personas, diciendo que la eficiencia es hacer las cosas correctamente, y la efectividad es hacer las cosas correctas. Sobre estas premisas nos alcanza algunas reflexiones filosóficas que inciden en la ciencia de la administración y en sus campos teóricos y prácticos.

La filosofía Drucker se expresa en categorías bien definidas que convergen en el seno de las organizaciones y permiten un proceso indefinido de gestión empresarial, donde se requiere una gran capacidad y experiencia de las personas que asumen la responsabilidad de llevar al éxito a la organización, bajo principios y valores verdaderamente filosóficos.

Veamos cuál es la filosofía de estos principios y valores según Drucker.

\section{SOBRE EL DINERO}

Dice que el dinero sigue al conocimiento. El dinero no es un problema; el problema es el liderazgo y la dirección. La rentabilidad no es un fin sino un factor determinante de la empresa. Si reflexionamos sobre estos conceptos se puede deducir que es el conocimiento el que ha llevado a la humanidad a sobreponerse a las grandes penurias para inexorablemente mejorar los niveles de calidad de vida.

En efecto, el dinero no es un problema porque su crecimiento está en función a los activos del conocimiento que se gestionan en las organizaciones, el cual se proyecta a los mercados-meta donde se efectúan las transacciones económicas y financieras que multiplican el crecimiento del dinero. Desde un punto de vista filosófico, son las organizaciones las que tienen que aprovechar las capacidades y las inteligencias de su gente para investigar y crear nuevos conocimientos que les permitan crecer y desarrollarse a un nivel competitivo.

La rentabilidad del dinero no puede considerarse como una finalidad del negocio; desde un punto de vista de gestión, la rentabilidad es uno de los principales objetivos de un empresario gerente y debe ser medida y evaluada, ya que lo que no se puede medir, no se puede gestionar.

\section{SOBRE LA ADMINISTRACIÓN}

La administración tiene que ver principalmente con la gente, no con técnicas ni procedimientos; su engranaje es lo que importa. El que toma las decisiones efectivas en realidad toma pocas decisiones.

Las tres preguntas claves para tomar decisiones son:

- ¿Cuál es nuestro negocio?

- ¿Quién es nuestro cliente?

- ¿Qué es lo que el cliente considera valor? 
Efectivamente, la administración es una ciencia que tiene un cuerpo teórico cada vez más complejo, como las demás ciencias sociales. También tiene un objeto de estudio orientado y referido a las organizaciones en todas sus formas, según su finalidad. Una administración o gestión exitosa depende de la capacidad de su gente, de la sinergia conjunta de los seres humanos, de la participación para solucionar no solamente los problemas sino los retos de la globalización de los mercados. El éxito organizacional se mide por la capacidad de su gente, por el conocimiento de los mismos, como dice Bill Gates: "A Microsoft no solamente ingresan los inteligentes, sino los suprainteligentes".

El negocio no está en los bienes o servicios que se ofrecen; el negocio está en su gente y, por lo tanto, se deben desarrollar sus capacidades y habilidades. Los clientes son cada vez más exigentes ante la inmensa cantidad de bienes y servicios; ellos tienen la libertad y el privilegio de elegir, pero esta libertad debe ser económica porque otro tipo de libertad no es una categoría de valor para lograr satisfacer una necesidad o un deseo. En la toma de decisiones se puede deducir cognitivamente que no guarda una relación de verdad absoluta así se haya tomado colectivamente con las personas más brillantes de la organización; por tanto, una decisión tiene un margen de incertidumbre y riesgo que puede desvirtuar el correcto proceso que encamine a materializar la visión propuesta.

\section{SOBRE EL CONOCIMIENTO}

Aceptamos el hecho de que el aprendizaje es un proceso de toda la vida para mantenernos actualizados respecto del cambio, y la tarea más apremiante es enseñar a la gente cómo aprender.

La esencia de la administración es hacer productivo el conocimiento que sólo existe en la aplicación (el conocimiento de acción a diferencia de sólo información). Si no es así estaremos limitados por la información que tengamos y por nuestra habilidad para procesar esa información.

Estas afirmaciones de Drucker invitan a los empresarios a desplegar enormes esfuerzos para crear nuevos conocimientos como una producción intelectual que se encuentra implícitamente en los niveles de competitividad y capacidad de la gente inmersa en la organización.
El conocimiento puede ser generado, descubierto, capturado, compartido, depurado, validado, transferido, adoptado, adaptado y aplicado. Las organizaciones no gestionan el conocimiento en sí, lo que se gestiona son los activos del conocimiento. El conocimiento es una abstracción mental del individuo que se consigue a través de la experiencia o experimentaciones. Para generar o crear conocimiento en las organizaciones se requiere tener las condiciones adecuadas; es decir, un ambiente apropiado con alto espíritu empresarial, tan igual como un filósofo requiere de un ambiente adecuado para transmontarse hasta los campos metafísicos. Asimismo, se necesitan los medio adecuados que incluyan los modelos, herramientas, procesos y la motivación de las personas para compartir y utilizar el conocimiento.

Uno de los recursos más escasos y valiosos en las organizaciones es el conocimiento que se encuentra en las personas que comparten toda la filosofía de la organización. Es preciso determinar qué tipo de conocimiento es el que se requiere para la gestión empresarial. Puede ser un conocimiento tácito, implícito o explícito o, en todo caso, los tres son indispensables para los negocios. En la concepción filosófica del conocimiento, cuando éste aparece y es exclusivo, es decir, que nunca ha existido, esto significa una reinvención para la empresa y el mundo. Se debe considerar que el conocimiento de hoy es la ignorancia del mañana. El conocimiento que se incorpora a la empresa se traslada a los productos o servicios que llevan al mercado un valor real para los clientes.

El conocimiento que se aplica en los negocios y en las industrias está sujeto a cambios rápidos y abruptos. Ante el agotamiento del petróleo en el mundo, el conocimiento estratégico, científico y técnico se aprovechará para descubrir nuevos sustitutos para el funcionamiento de los negocios que consumen energía proveniente de los recursos naturales.

\section{SOBRE EL INDIVIDUO}

Es importante conocer las fortalezas de los individuos y repotenciar estas cualidades en forma continua; esto es lo que se necesita hacer con la gente para lograr su pleno desarrollo. Cada persona quiere y debe ser recordada por algún hecho descollante que haya beneficiado a la organización o haya cambiado su propia filosofía. Cuando se habla de la gente tenemos que preocuparnos por 
sus capacidades en términos de productividad desprendida del nuevo conocimiento. Además se tiene que aplicar el nuevo conocimiento al conocimiento como factor fundamental para el avance e incremento de la productividad.

En las enseñanzas que nos dejó Peter Drucker de cómo invertir en la gente y en el conocimiento, nos planteó las siguientes consideraciones:

- Dada su estrategia general de negocios y el alcance de la oferta de su producto o servicio ¿en cuáles capacidades centrales quiere invertir?

- ¿Qué papel juega el conocimiento en el valor que ofrece a sus clientes y en sus capacidades centrales?

- ¿Cómo y hasta qué alcance está invirtiendo en atraer y desarrollar a su gente en la base del conocimiento?

"El amor al conocimiento es el fin de la sabiduría", decía Confucio. Debemos comprender que los hombres con conocimientos superiores son los llamados a desempeñar cargos importantes con mucha discreción, tolerancia, y con visión de futuro. El hombre de empresa debe ser abierto y sereno, digno pero no orgulloso, permanecer siempre en lo correcto, firme en sus decisiones, liberal con las opiniones de los demás, tener vocación de servicio, mantener el prestigio personal.

Un hombre con altos conocimientos puede dirigir con más desenvolvimiento gerencial a una organización cualquiera sea su tamaño, pero con la única condición de tener a su lado a colaboradores que tengan valor y se enfrenten a los riesgos que siempre presentan los negocios. Una gerencia es exitosa cuando simplemente se ponen las cosas en su lugar y cuando se predica con el ejemplo. Confucio decía: "El carácter del gobernante es como el viento, y el carácter de la gente común es cómo la hierba, y la hierba se inclina en dirección del viento".

\section{INVERTIR EN EL CONOCIMIENTO Y EN LA GENTE}

De acuerdo a su filosofía de la gerencia, Peter Drucker dice: "Lo que diferencia a las organizaciones es si estas pueden hacer que la gente común realice cosas no comunes". Este planteamiento es una dinámica en las organizaciones, y para esto es importante formular y reformular las siguientes preguntas:
- ¿Quién es la gente correcta para tu organización?

- ¿Estás proporcionando a tu gente los medios para alcanzar su efectividad máxima y contribuir al éxito de la organización?

- ¿Tu estructura y procesos institucionalizan el respeto para la gente y la inversión en el capital humano?

- ¿El conocimiento y el acceso al conocimiento están incorporados en tu forma de hacer negocios?

- ¿Cuál es tu estrategia para invertir en la gente y en el conocimiento?

"El verdadero secreto para lograr el éxito de la empresa es su excepcional habilidad para convertir a sus trabajadores en altamente productivos, motivados y altamente conscientes de la calidad al reconocer el potencial, la dignidad y el valor del individuo".

Estas expresiones de Peter Drucker depositan en el conocimiento el recurso más valioso de las organizaciones, del cual depende el futuro de la empresa.

En mi preocupación por desarrollar una filosofía de la administración quiero proponer algunos enfoques que deben considerarse como puntos de partida para crear una teoría filosófica de la administración:

- En las organizaciones, el gerente debe cultivar una ejemplar conducta personal.

- Reconocer, distinguir, premiar y honrar a las personas dignas de las organizaciones.

- Guardar el respeto y el afecto a todas las personas que cumplan con sus deberes y responsabilidades.

- Despertar el interés y promover el bienestar de todos los individuos de la organización.

- Convertirse en un paradigma a seguir por todos, enseñando y aprendiendo de los demás.

- Invertir para lograr el pleno desarrollo de las personas y así lograr nuevos conocimientos que permitan el éxito de la organización.

- Ser benevolente, tolerante y facilitador con los clientes de la organización; de ellos depende parte del éxito. 


\section{BIBLIOGRAFÍA}

Boyett, Joseph y Boyett, Jimmie. (2000). Lo mejor de los Gurús. Buenos Aires, Editorial Gestión.

Canals, Agusti. (2000). Gestión del conocimiento. Barcelona, Editorial Gestión 2000.

Drucker, Peter. (1992). La Gerencia. Buenos Aires, Editorial El Ateneo.

Lafley, A.G. (2007). Enseñanzas de Peter Drucker. Buenos Aires, Editorial Mc Graw Hill.
Martinez Caraballo, Noemí. (2006). Gestión del conocimiento. Madrid, Harvard Business Review.

Nonaka, Kujiro y Takeuchi, Hirotaka. (2004). La organización creadora de conocimiento. México, Editorial Oxford University.

Yutang, Lin. (1999). La sabiduría de Confucio. Buenos Aires, Editorial Siglo XX. 